\begin{tabular}{|c|c|}
\hline Title & Collimator for V ariable Sensitivity and Spatial Resolution Without the Need for Exchange \\
\hline Author(s) & $\begin{array}{l}\text { Kubo, Naoki; T suchiya, Katsutoshi; Shiga, Tohru; Kojima, Shinichi; Suzuki, A tsuro; Ueno, Y uichiro; Kobashi, Keiji; } \\
\text { Tamaki, Nagara }\end{array}$ \\
\hline Citation & $\begin{array}{l}\text { IEEE Transactions on Nuclear Science, 61(5), 2489-2493 } \\
\text { https://doi.org/10.1109/ NS.2014.2333748 }\end{array}$ \\
\hline Issue Date & $2014-0808$ \\
\hline Doc URL & http:/hdl.handle.net/2115/64691 \\
\hline Rights & $\begin{array}{l}\text { C } 2014 \text { IEEE. Personal use of this material is permitted. Permission from IEEE must be obtained for all other uses, in } \\
\text { any current or future media, including reprinting/republishing this material for advertising or promotional purposes, } \\
\text { creating new collective works, for resale or redistribution to servers or lists, or reuse of any copyrighted component of } \\
\text { this work in other works. }\end{array}$ \\
\hline Tyре & article (author version) \\
\hline File Information & TNS-00027-2014.R1.pdf \\
\hline
\end{tabular}

Instructions for use 


\title{
Collimator for Variable Sensitivity and Spatial Resolution Without the Need for Exchange
}

\author{
Naoki Kubo, Katsutoshi Tsuchiya, Tohru Shiga, Shinichi Kojima, Atsuro Suzuki, Yuichiro Ueno, \\ Keiji Kobashi, and Nagara Tamaki
}

\begin{abstract}
A new design of collimator is proposed that has variable sensitivity and spatial resolution, eliminating the need for exchanging collimators in a gamma camera. Using Monte Carlo simulations, the present article evaluates the shielding of undesirable gamma rays in a parallel-hole collimator. It consists of a number of layers of rectangular holes. These layers consist of alternately stacked fixed and movable collimators. In high-resolution mode, the movable collimators are shifted by half the aperture pitch along the diagonal direction. The first collimator (type A) has 50 layers with fixed thicknesses of $\mathbf{1 . 2} \mathbf{~ m m}$. The second collimator (type B) has 25 layers with a thickness of $1.0 \mathrm{~mm}$ on the object side and 25 layers with a thickness of $1.4 \mathrm{~mm}$ on the opposite side. The third collimator (type $\mathrm{C}$ ) has 20 layers with non-uniform thicknesses. The ratios of the maximum artificial peak to the main-peak are calculated for point-source responses. The ratios for types $\mathrm{A}, \mathrm{B}$, and $\mathrm{C}$ collimators are $\mathbf{0 . 7 8}$, 0.08 , and 0.03 , respectively. The same performance for shielding undesirable gamma rays is achieved in the type $C$ collimator as for a conventional collimator.
\end{abstract}

Index Terms-Collimators, High-resolution, Monte Carlo simulation.

\section{INTRODUCTION}

A collimator is a key mechanical component of a gamma camera [1] for radioactive source imaging for medical applications. It provides an abundance of information for evaluation of the severity of diseases, prognosis, and therapeutic effects [2]. There needs to be a one-to-one correspondence between the direction of emission of gamma rays from a radioactive source and its point of detection [3]. For this purpose, one type of parallel-hole collimator is composed of many precisely aligned holes (channels) formed of lead or tungsten. The collimator allows only those gamma rays traveling directly along the long axis of the holes to pass. Gamma rays traveling in other directions are absorbed by the septa between the holes. Collimators essentially determine the spatial resolution and sensitivity of a gamma camera system. Therefore, several collimators are used for imaging with

N. Kubo is with the Office of Health and Safety, Hokkaido University, Sapporo-shi, Hokkaido, Japan.

K. Tsuchiya, S. Kojima, A. Suzuki, Y. Ueno, and K. Kobashi are with the Central Research Laboratory, Hitachi Ltd., Hitachi-shi, Ibaraki-ken, Japan.

T. Shiga and N. Tamaki are with the Graduate School of Medicine, Hokkaido University, Sapporo-shi, Hokkaido, Japan. varying sensitivity and spatial resolution. A high-resolution collimator has a large number of small holes, and therefore has low sensitivity. This kind of collimator is used when the total number of emitted gamma rays is large enough to tolerate the low sensitivity. That depends on the level of radioactivity and the image acquisition time. A high-sensitivity collimator has large holes and consequently low spatial resolution. It is used for dynamic imaging when short frame times are required and the reduced spatial resolution is acceptable [4]. In addition, handheld cameras equipped with parallel-hole collimators have emerged in medical fields such as surgery and pre-surgery sentinel node and thyroid scintigraphy [5-6]. For such applications, collimator exchange is necessary. If this exchange process could be eliminated, the risk of collimator damage in an accidental fall and the time required for the exchange would be avoided. This contributes to safety. We propose a new collimator design with variable sensitivity and spatial resolution that eliminates the need for exchange. Using Monte Carlo simulations, the present article evaluates the shielding of unwanted gamma rays in the collimator.

\section{DESIGN CONCEPT}

The design is a parallel-hole collimator consisting of a number of layers of multiple rectangular holes, sketched in Fig. 1.

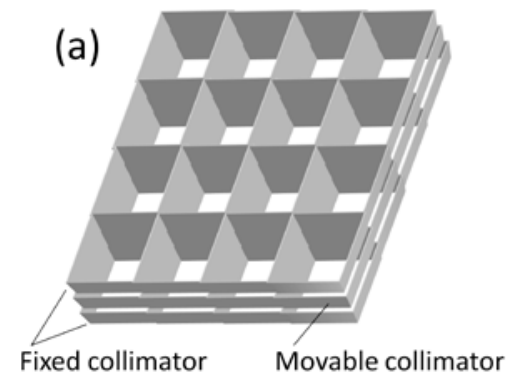

(b)
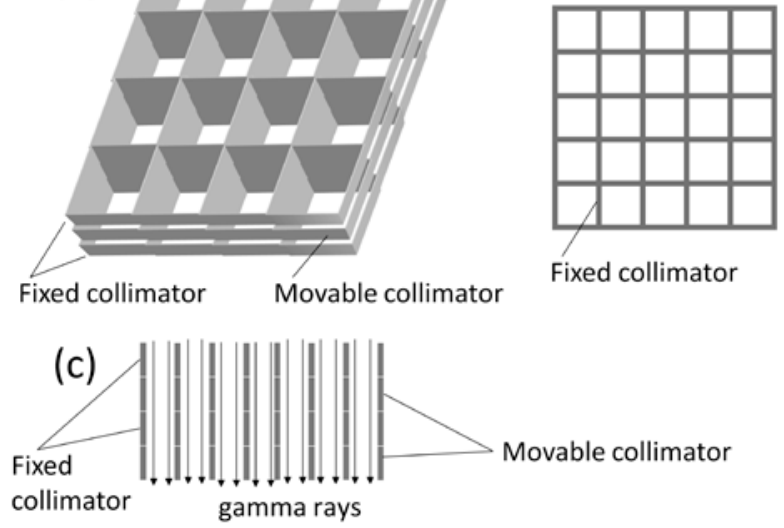

Fig. 1. (a) Schematic of the proposed collimator. The layers correspond to fixed and movable collimators that are alternately stacked. In normal mode, the apertures are aligned along the axial direction of the holes. (b) Top view of the collimator in normal mode. The movable collimators overlap the fixed collimators. (c) Side view of the collimator in normal mode. The parallel holes only allow the passage of gamma rays traveling directly along the long axis of the holes. 
These layers represent alternately stacked fixed and movable collimators. In the normal mode, the fixed and movable apertures are aligned along the axial direction of the holes, as in a conventional parallel-hole collimator.

In high-resolution mode, the movable collimators are shifted by half the aperture pitch along the diagonal direction, as indicated in Fig. 2. Consequently, the aperture area is decreased by a factor of four. The septa in this mode have gaps similar to a wire-mesh collimator [7-12]. During use, the margin and sides of the collimator are shielded with a cover.
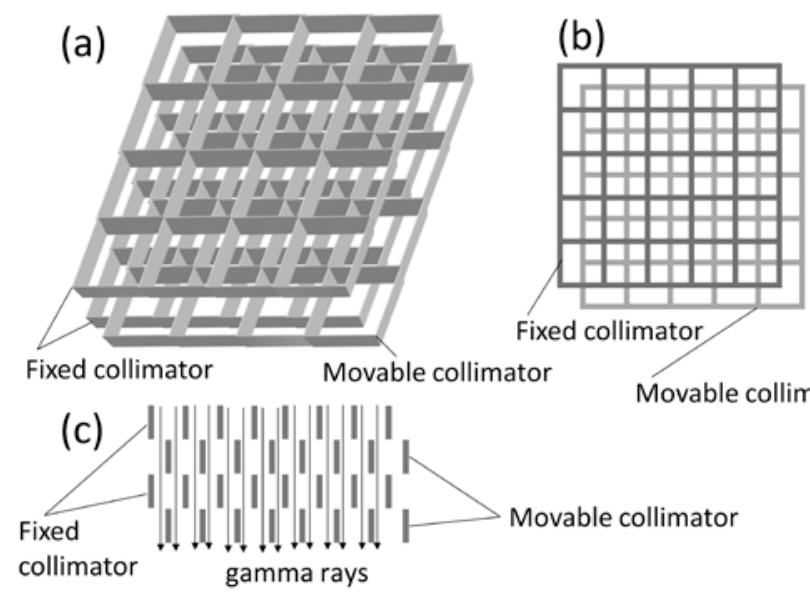

Fig. 2. (a) Diagram of the collimator in high-resolution mode. (b) Top view, showing that the movable collimators are shifted by half the aperture pitch along the diagonal. (c) The side view indicates that the septa now have gaps similar to those of a wire-mesh collimator.

\section{METHODS}

\section{A. Collimator shape in normal mode}

Assume a collimator with a field of view (FOV) of $240 \times 240$ $\mathrm{mm}^{2}$ for a small head gamma camera. The holes, rectangular in shape, had an area of $3.0 \times 3.0 \mathrm{~mm}^{2}$, a septal thickness of 0.2 $\mathrm{mm}$, and a length of $60 \mathrm{~mm}$. The geometric efficiency of the collimator was given by the formula:

$$
g=\left[\frac{K \cdot d^{2}}{a(d+t)}\right]^{2},
$$

where $g$ is the number of gamma rays that pass through the holes divided by the total number emitted by the subject. The width of a hole is $d$, the length is $a$, the septal thickness is $t$, and $K=0.282$ for square holes [13]. The geometric efficiency of the collimator in normal mode was $1.8 \times 10^{-4}$. This performance was similar to that of a general-purpose collimator.

\section{B. Collimator shape in high-resolution mode}

Three collimator configurations were considered. The first (type A) was a collimator with 50 layers having fixed thicknesses of $1.2 \mathrm{~mm}$. The second collimator (type B) had 25 layers with thicknesses of $1.0 \mathrm{~mm}$ on the object side, and 25 layers with thicknesses of $1.4 \mathrm{~mm}$ on the opposite side. The third configuration (type C) had 20 layers with thicknesses listed in Table 1 determined by a computerized random number generator. Each layer thickness (lt) was then calculated from the formula:

$$
I t_{i}=3.00+1.50 r_{i},
$$

where $-1 \leq r_{i} \leq 1$ are random number from the uniform distribution. Each thickness was normalized to $60-\mathrm{mm}$ hole length of the collimator.

TABLE I

NON-UNIFORM LAYER THICKNESSES FOR THE TYPE C COLLIMATOR

\begin{tabular}{cc}
\hline \hline Layer Number & Thickness $(\mathrm{mm})$ \\
\hline 1 & 4.25 \\
2 & 2.36 \\
3 & 1.79 \\
4 & 1.56 \\
5 & 2.83 \\
6 & 3.67 \\
7 & 2.81 \\
8 & 2.97 \\
9 & 2.06 \\
10 & 2.10 \\
11 & 4.33 \\
12 & 3.69 \\
13 & 2.90 \\
14 & 3.80 \\
15 & 2.51 \\
16 & 4.38 \\
17 & 4.11 \\
18 & 2.97 \\
19 & 2.41 \\
20 & 2.50 \\
\hline \hline
\end{tabular}

The layers correspond to fixed and movable collimators that were alternately stacked. The movable collimators were shifted by half the aperture pitch along the diagonal direction. The rectangular holes had an area of $1.4 \times 1.4 \mathrm{~mm}^{2}$, as illustrated in Fig. 3. The geometric efficiency in high-resolution mode was $3.4 \times 10^{-5}$.

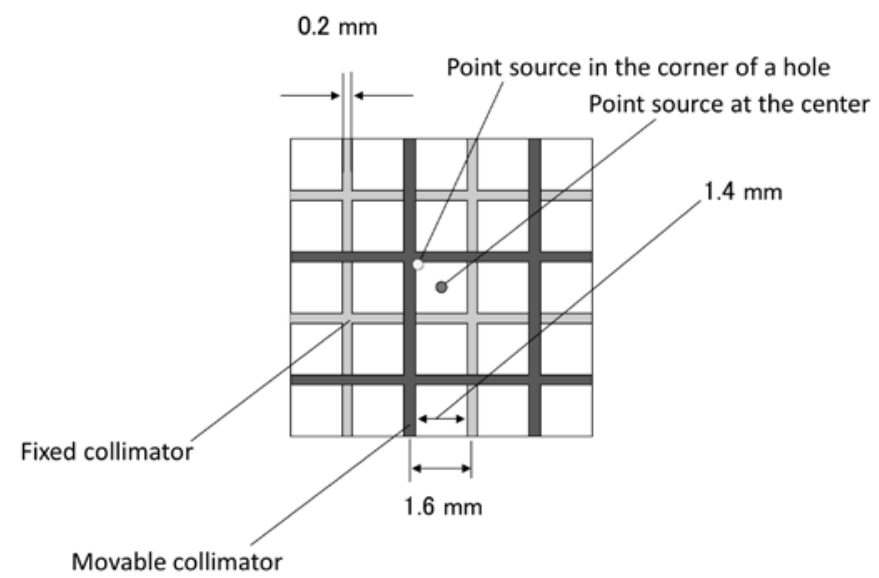

Fig. 3. Geometric configuration of the collimator in high-resolution mode.

\section{Simulation using a point source}

The performance of the proposed collimator was confirmed by Monte Carlo simulations, using the EGS4 computer code. The number of gamma rays was 10 million, emitted from a point source of ${ }^{99 \mathrm{~m} T c}(140 \mathrm{keV})$ located at the center of the 
collimator. The distance from the source to the collimator surface was $0.001 \mathrm{~cm}$. The collimator material was lead. The efficiency of the detector was assumed to be $100 \%$. With regard to the interactions of photons with the material, Compton scattering, coherent scattering, and the photoelectric effect were all included. In addition, a simulation was performed with a point source located in the corner of a hole of the type $\mathrm{C}$ collimator.

\section{Analysis of artificial peaks}

To evaluate the shielding of undesirable gamma rays, the ratio of the maximum artificial peak to the main peak was calculated.

\section{E. Spatial resolution of the collimator having non-uniform layer thicknesses}

For source-to-collimator distances of $0.001,10$ and $20 \mathrm{~cm}$, simulations were performed with a point source located at the center of the type $\mathrm{C}$ collimator. Full width at half maximum (FWHM) and full width at tenth maximum (FWTM) were measured directly from the profiles using linear interpolation.

\section{RESUlTS}

The point-source responses at the center of types A, B, and C collimators in high-resolution mode are plotted in Figs. 4, 5 and 6 , respectively. Fig. 7 shows the point-source response in the corner of a hole of type $\mathrm{C}$. The ratios of the maximum artificial peak to the main peak for types $\mathrm{A}, \mathrm{B}$, and $\mathrm{C}$ collimators with a FOV of $240 \times 240 \mathrm{~mm}^{2}$ were $0.78,0.08$, and 0.03 , respectively.

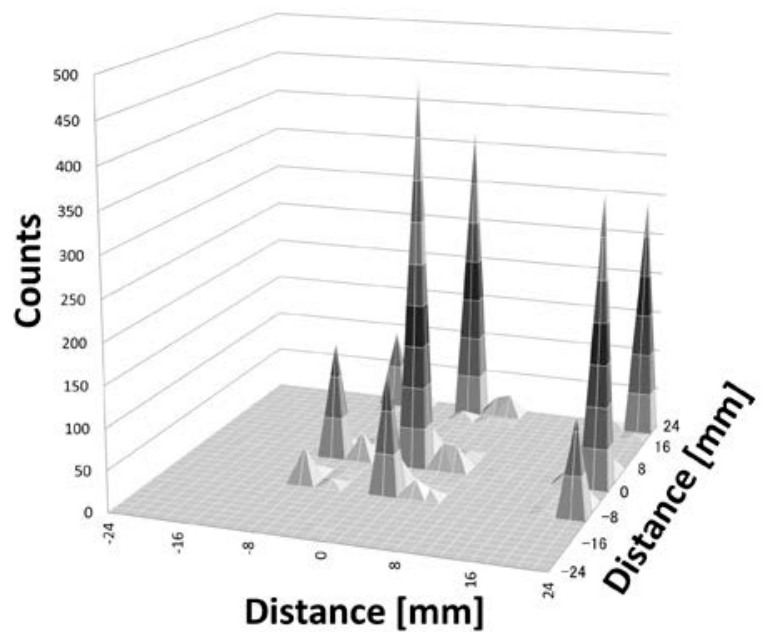

Fig. 4. Point-source response of a type A collimator having fixed layer thicknesses. This graph plots the center of a $48 \times 48 \mathrm{~mm}^{2}$ area. A point source was located at the center of the collimator.

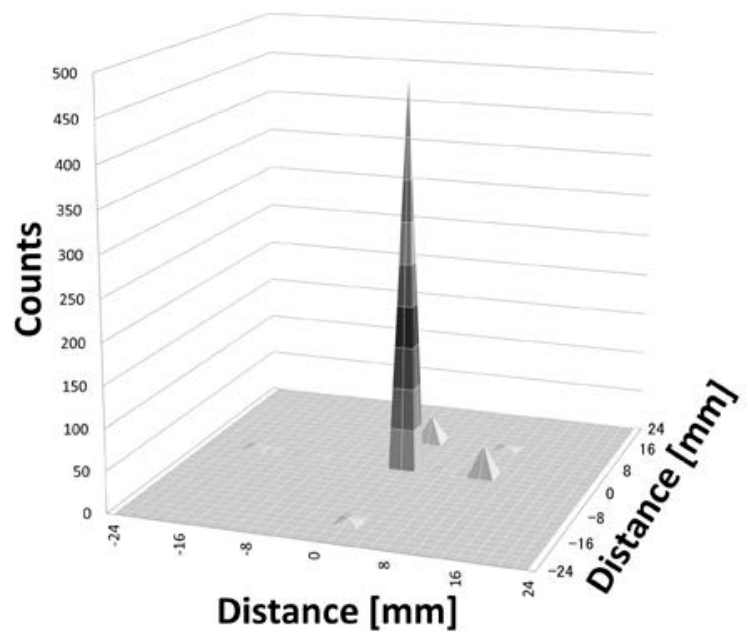

Fig. 5. Point-source response of a type B collimator. It has 25 layers with thicknesses of $1.0 \mathrm{~mm}$ on the object side, and 25 layers with thicknesses of 1.4 $\mathrm{mm}$ on the opposite side.

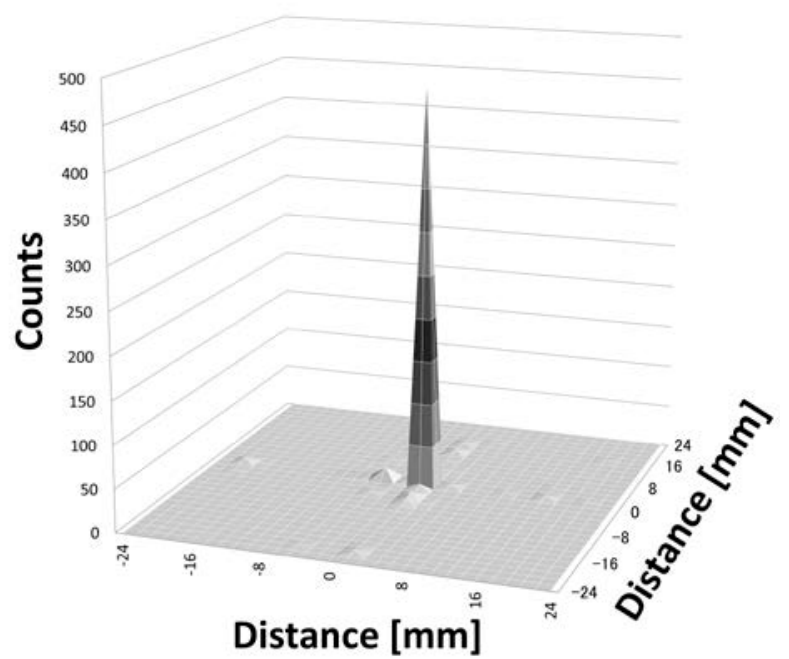

Fig. 6. Point-source response of a type $\mathrm{C}$ collimator having non-uniform layer thicknesses.

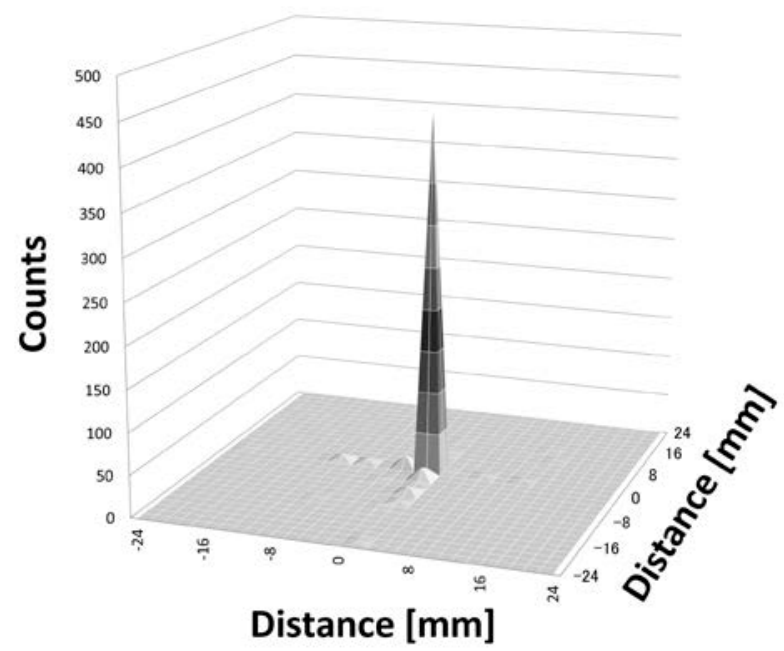

Fig. 7. Point-source response of a type $C$ collimator having non-uniform layer thicknesses. A point source was located in the corner of a hole. 
The widths of the main peak under the point source (source to collimator distance of $0.001 \mathrm{~cm}$ ) were nearly equal to the hole size in high-resolution mode. In Fig. 8, FWHM and FWTM increased monotonically with increasing source-to-collimator distance.

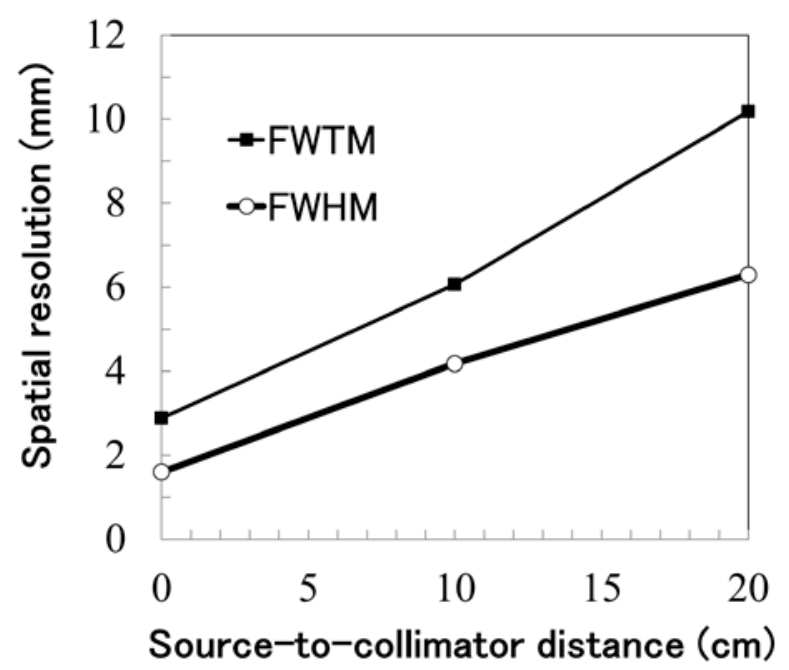

Fig. 8. Geometric resolution of a type $\mathrm{C}$ collimators with source-to-collimator distance. $\mathrm{FWHM}=$ full width at half maximum. FWTM $=$ full width at tenth maximum.

\section{DISCUSSION}

A new collimator has been designed for variable sensitivity and spatial resolution. The optimum thicknesses of this collimator have been evaluated. The design eliminates the need for collimator exchange in a gamma camera, thereby avoiding collimator damage, exchange time, and the need to move the camera head. In sentinel node surgery using a handheld camera, radiopharmaceutical uptake is used to search in the general-purpose sensitivity mode initially. Next, a high-resolution mode is employed for detailed image acquisition, without moving the camera head from the target field. The radioactive dynamic range is broad in dynamic acquisition with bolas injection using a conventional gamma camera [14]. Therefore, some count loss can occur when measuring high radioactivity using a conventional collimator. In contrast, with a variable sensitivity and resolution collimator, the high-resolution (lower sensitivity) mode can be used in high radioactivity to prevent any count loss.

To reduce the weight, the wire-mesh collimator was introduced. It is made from a series of wire grids [7-12]. Generally, a conventional collimator is overspecified in terms of its path length in the material composing the collimator [8]. Thus, a wire-mesh collimator successfully competes with a parallel-hole collimator. The newly proposed multilayer collimator in high-resolution mode is similar to the wire-mesh collimator. As a result, this study is consistent with previous reports [7-12].

The septal penetration is set to 0.05 in most conventional collimators [15]. Penetrating gamma rays significantly reduce contrast and image quality. In this study, artificial peaks should include gamma rays transmitted through septa and also traveled along free space without encountering septa. Artificial peaks in high-resolution mode, as seen in Fig. 6 and 7, in the non-uniform thickness condition were less than 0.05 . The effect of artificial peaks should be similar to that of penetrating gamma rays in conventional collimators. The source positional-dependence for shielding undesirable gamma rays was not observed in Fig. 6 and 7. Thus, the same performance was thus achieved for shielding undesirable gamma rays by type $\mathrm{C}$ as by a conventional collimator.

For a fixed layer thickness of $1.2 \mathrm{~mm}$, several artificial peaks occur. As seen in Fig. 9(a), the path $\beta$ arises in addition to the normal path $\alpha$. However, this is not the case for collimators with non-uniform thicknesses, as Fig. 9(b) shows.
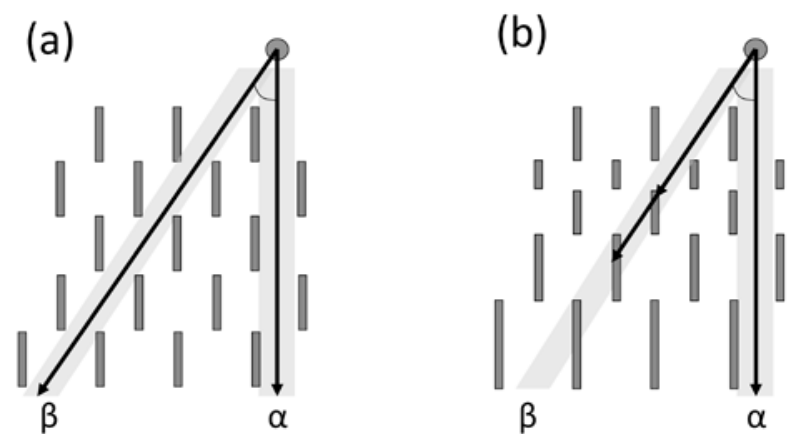

Fig. 9. (a) Ray paths for a collimator having uniform layer thicknesses. Path $\beta$ causes an artificial peak in the point-source response. Path $\alpha$ is desirable. (b) Ray paths for a collimator having non-uniform thicknesses. Path $\beta$ no longer arises.

No space was left between layers in this simulation. In actuality, spaces between layers are necessary. However, these spaces are small enough that the results of the simulations are not expected to differ much from the actual conditions.

In the present study, multiple rectangular apertures were used because pixel-based gamma cameras have emerged for imaging [16-19]. With this type of camera, pixel matching to the collimator holes can be achieved using a collimator with multiple rectangular apertures.

In high-resolution mode, the movable collimators are shifted by half the aperture pitch in two directions. The mechanism can be as simple as sliding, and can be realized by an electric relay switch or by manual sliding on rails with a stopper. A multileaf collimator for radiotherapy could use these movable collimators.

The collimator manufactured by Acrorad Co. Ltd. (Tokyo, Japan) had a thickness of $10 \mathrm{~mm}$, which comprised a stack of 100 pieces of 0.1-mm-thick tungsten mesh sheets, each having $1.2 \times 1.2 \mathrm{~mm}^{2}$ square openings [20]. Thus, a layered collimator as type $\mathrm{A}, \mathrm{B}$ and $\mathrm{C}$ will be realized by the use of tungsten mesh sheets.

The shielding performance of a type $\mathrm{C}$ collimator in high-resolution mode was similar to that of a conventional collimator. The relation between the spatial resolution and the source-to-collimator distance was similar to that for a conventional collimator in Fig. 8 [21]. That relation has been previously reported for a wire-mesh collimator [8]. The results are acceptable for clinical applications. Equally, the performance of a type $\mathrm{C}$ collimator would be acceptable. 
In the present study, only Monte Carlo simulations were carried out. The simulations were limited and that the possibility of significant artificial peaks still exists. Further simulations would be necessary. However, in the future, we plan to experimentally evaluate the performance of the newly designed collimator.

\section{CONCLUSIONS}

A collimator with variable sensitivity and spatial resolution has been designed that eliminates the need for exchanging collimators. The performance of this collimator in highresolution mode has been confirmed by Monte Carlo simulations. The results are suitable for clinical applications.

\section{REFERENCES}

[1] L. Jansen, O. E. Nieweg, A. E. Kapteijn, R. A. Valdés Olmos, S. H. Muller, C. A. Hoefnagel, and B. B. Kroon, "Reliability of lymphoscintigraphy in indicating the number of sentinel nodes in melanoma patients," Ann. Surg. Oncol., vol. 7, no. 8, pp. 624-630, 2000.

[2] S. Yamashina and J. Yamazaki, "Neuronal imaging using SPECT," Eur. J. Nucl. Med. Mol. Imaging, vol. 34, no. 6, pp. 939-950, 2007.

[3] S. C. Moore, K. Kouris, and I. Cullum, "Collimator design for single photon emission tomography,” Eur. J. Nucl. Med., vol. 19, no. 2, pp. 138$150,1992$.

[4] T. Jonasson, "Adaptation of new electronics and conversion of the camera to a demonstrator in medical imaging," in Revival of a Gamma Camera, Stockholm: Master of Science Thesis, Royal Institute of Technology, 2003, pp. 1-43.

[5] J. Ortega, J. Ferrer-Rebolleda, N. Cassinello, and S. Lledo, "Potential role of a new hand-held miniature gamma camera in performing minimally invasive parathyroidectomy,” Eur. J. Nucl. Med. Mol. Imaging, vol. 34, no. 2, pp. 165-169, 2007.

[6] K. Kerrou, S. Pitre, C. Coutant, R. Rouzier, P. Y. Ancel, C. Lebeaux, V. Huchet, F. Montravers, O. Pascal, M. A. Duval, F. Lefebvre, L. Menard, S. Uzan, Y. Charon, and E. Barranger, "The usefulness of a preoperative compact imager, a hand-held gamma-camera for breast cancer sentinel node biopsy: final results of a prospective double-blind, clinical study," $J$. Nucl. Med., vol. 52, no. 9, pp. 1346-1353, 2011.

[7] K. Ogawa and J. Kato, “Collimator design for single photon emitter," IEEE Nucl. Sci. Symp. Conf. Rec., vol. 2, pp. 760-763, 2002.

[8] K. Ogawa and J. Kato "Wired collimator for single photon emitter," IEEE Trans. Nucl. Sci., vol. 50, no. 5, pp. 15361540, 2003.
[9] M. I. Saripan, M. Petrou, and K. Wells, "Design of a wire-mesh collimator for gamma cameras,” IEEE Trans. Biomed. Eng., vol. 54, no. 9, pp. 1598-1612, 2007.

[10] M. I. Saripan, W. H. M. Saad, S. Hashim, R. Mahmud, Nordin, and M. A. Mahdi, "Monte Carlo Simulation on Breast Cancer Detection Using Wire Mesh Collimator Gamma Camera,” IEEE Trans. Nucl. Sci., vol. 56, no. 3, pp. 1321-1324, 2009.

[11] R. E. Roslan, W. H. M. Saad, M. I. Saripana, S. Hashimb, and W. -S. Choongc, "The performance of a wire mesh collimator SPECT camera for different breast volumes in prone position," Nucl. Instrum. Methods Phys. Res. A, vol. 619, pp. 385-387, 2010.

[12] W. H. M. Saad, R. E. Roslan, M. A. Mahdi, W. -S. Choong, E. Saion, and M. I. Saripan, "Monte Carlo design of optimal wire mesh collimator for breast tumor imaging process," Nucl. Instrum. Methods Phys. Res. A, vol. 648, pp. 254-260, 2011.

[13] H. O. Anger, "Scintillation camera With multichannel collimators," $J$. Nucl. Med., vol. 5, pp. 515-531, 1964.

[14] R. J. Gibbons, K. L. Lee, F. Cobb, and R. H. Jones, "Ejection fraction response to exercise in patients with chest pain and normal coronary arteriograms," Circulation, vol. 64, no. 5, pp. 952-957, 1981.

[15] A. L. Weinmann, C. B. Hruska, and M. K. O'Connor, "Design of optimal collimation for dedicated molecular breast imaging systems," Med. Phys., vol. 36, no. 3, pp. 845-856, 2009.

[16] M. B. Williams, M. B. Williams, A. R. Goode, V. Galbis-Reig, S. Majewski, A. G. Weisenberger, and R. Wojcik, "Performance of a PSPMT based detector for scintimammography," Phys. Med. Biol., vol. 45, no. 3, pp. 781-800, 2000.

[17] N. Kubo, S. Zhao, Y. Fujiki, A. Kinda, N. Motomura, C. Katoh, T. Shiga, H. Kawashima, Y. Kuge, and N. Tamaki, "Evaluating performance of a pixel array semiconductor SPECT system for small animal imaging," Ann. Nucl. Med., vol. 19, no. 7, pp. 633-639, 2005.

[18] C. Robert, G. Montémont, V. Rebuffel, I. Buvat, L. Guérin, and L. Verger, "Simulation-based evaluation and optimization of a new CdZnTe gamma-camera architecture (HiSens),” Phys. Med. Biol., vol. 55, no. 9, pp. 2709-2726, 2010.

[19] W. Siman and S. C. Kappadath, "Performance characteristics of a new pixelated portable gamma camera,” Med. Phys., vol. 39, no. 6, pp. 34353444, 2012.

[20] M. Tsuchimochi, H. Sakahara, K. Hayama, M. Funaki, R. Ohno, T. Shirahata, T. Orskaug, G. Maehlum, K. Yoshioka and E. Nygard, “A prototype small CdTe gamma camera for radioguided surgery and other imaging applications," Eur. J. Nucl. Med. Mol. Imaging, vol. 30, no. 12, pp. 1605-1614, 2003.

[21] J. Zeintl, A. H. Vija, A. Yahil, J. Hornegger and T. Kuwert, "Towards quantitative SPECT: Error estimation of SPECT OSEM with 3D resolution recovery, attenuation correction and scatter correction," IEEE Nucl. Sci. Symp. Conf. Rec., pp. 4106-4111, 2008. 